MONOGRÁFICO

Didáctica de la Lengua:

Un cruce de caminos

\title{
APRENDIZAJE DEL VOCABULARIO: UNA EXPERIENCIA FORMATIVA EN CUARTO CURSO DE EDUCACIÓN PRIMARIA
}

\author{
Recepción: 29/09/2017 | Revisión: 29/11/2017 | Aceptación: 27/12/2017
}

\author{
Ana TEBEROSKY CORONADO \\ Aneska ORTEGA BLANCO \\ Universitat de Barcelona \\ Universitat de Barcelona \\ ateberosky@ub.edu \\ aortegbl7@gmail.com
}

Resumen: En este artículo se reflexiona sobre el aprendizaje del vocabulario académico en niños escolares y se presenta un diseño de intervención formativa que muestra una propuesta de enseñanza para ayudar a los alumnos en dicho aprendizaje. La reflexión se hace sobre el papel del vocabulario en el proceso de enseñanza-aprendizaje-comprensión de un contenido específico y sobre su relación con las posibles dificultades de lectura debidas al uso del lenguaje académico para expresar un contenido disciplinar. La intervención tiene como objetivo orientar las actividades y procedimientos de aprendizaje de los alumnos al seleccionar y organizar el vocabulario académico y al comparar la composición morfológica de las palabras y su historia etimológica. Dicha experiencia de intervención se realizó en el $4^{\circ}$ curso de Primaria de una escuela pública de Barcelona sobre el tema de los fenómenos atmosféricos.

Palabras clave: aprendizaje de vocabulario; experiencia formativa; morfología.

\section{VOCABULARY LEARNING: A FORMATIVE EXPERIENCE IN FOURTH YEAR OF PRIMARY EDUCATION}

\begin{abstract}
This article analyzes the learning of academic vocabulary in school children and presents a design of formative intervention that shows a teaching proposal to help students in such learning. The reflection is based on the role of vocabulary in the teaching-learning-comprehension process of a specific content and on its relationship with the possible difficulties of reading due to the use of the academic language to express a disciplinary content. The formative intervention aims to guide the learning activities and procedures of students in selecting and organizing the academic vocabulary and comparing the morphological composition of words and their etymological history. The study included 28 students in their $4^{\text {th }}$ year of primary school and the experiment of intervention was carried out in a public school in Barcelona on the subject of atmospheric phenomena.
\end{abstract}

\section{Keywords:}

vocabulary learning; formative experience; morphology.

\section{APRENENTATGE DEL VOCABULARI: UNA EXPERIENNCIA FORMATIVA EN EL OUART CURS D'EDUCACIÓ PRIMÀRIA}

Resum: En aquest article es reflexiona sobre l'aprenentatge del vocabulari acadèmic en nens en edat escolar i es presenta un disseny d'intervenció formativa que mostra una proposta d'ensenyament per ajudar els alumnes en aquest aprenentatge. La reflexió es fa sobre el paper del vocabulari en el procés d'ensenyament-aprenentatge-comprensió d'un contingut específic i sobre la seva relació amb les possibles dificultats de lectura degudes a l'ús del llenguatge acadèmic per expressar un contingut disciplinar. La intervenció té com objectiu orientar les activitats i procediments d'aprenentatge dels alumnes en seleccionar i organitzar el vocabulari acadèmic $i$ en comparar la composició morfolòica de les paraules $i$ la seva historia etimològica. Aquesta experiència d'intervenció es va realitzar en el 4t curs de Primària d'una escola pública de Barcelona sobre el tema dels fenòmens atmosfèrics.

\section{Paraules clau:}

aprenentatge de vocabulari; experiència formativa; morfologia. 


\title{
1. El vocabulario de los textos académicos, su aprendizaje y enseñanza
}

Los alumnos de $4^{\circ}$ curso de Primaria suelen tener que leer y comprender textos sobre temas académicos como el siguiente extracto, que es la traducción de un texto original en catalán.

\begin{abstract}
«El tiempo atmosférico tiene tres elementos: la temperatura, las precipitaciones y el viento. La atmósfera es una mezcla de gases que rodea la tierra y la temperatura atmosférica es el grado de calor del aire... Por efecto del calor del sol, el agua de los ríos, de los lagos y del mar se evapora, la evaporación produce precipitaciones... El viento es una corriente producida por masas de aire que se mueven rápidamente. Cuando la atmósfera se calienta o se enfría, se producen estas corrientes de aire... La meteorología es la ciencia que estudia la atmósfera y los fenómenos que se producen, como si llueve, hace aire o calor».
\end{abstract}

Este texto se usó para informar sobre el tema «Fenómenos atmosféricos» del currículo escolar. En este extracto, representativo de muchos textos escolares, se puede apreciar que su autor proporciona pistas o ayudas para su comprensión, entre ellas incluye definiciones de conceptos y explicaciones de procesos para introducir conocimientos nuevos. Se define atmósfera, temperatura atmosférica, viento y meteorología porque se trata de términos que carecen de referentes tangibles, es decir son nombres de conceptos complejos. Y se explica la causa de los procesos que no son acciones simples: las precipitaciones y las corrientes de aire. A pesar de ello, el texto resulta difícil para los alumnos de $4^{\circ}$ curso. La dificultad reside, según Nippold y Sun (2008), tanto en el contenido de información exigente con respecto a la base de conocimientos necesarios para comprenderlo, como en el lenguaje académico poco frecuente de formulación de definiciones y de explicaciones en forma entrelazada que se apoyan y dependen de la información verbal dada en el propio texto.

\subsection{Características del texto académico}

Los estudios recientes que analizan las características del lenguaje académico en los textos (Car-lisle, 2003; Chung y Nation, 2003; Fang, 2008; 2012; Harmon, Hedrick, y Wood, 2005; Nagy y Anderson, 1984; Nagy y Townsend, 2012; Nippold, 2007; Snow y Uccelli, 2009) sostienen que usan un lenguaje especializado en transmitir información técnica, abstracta y densa (de elevada concentración) sobre fenómenos de diferentes ámbitos disciplinares. El lenguaje académico es técnico en relación al tema que trata el texto y es diferente del lenguaje de la conversación casual. Según varios autores (Biber, 2006; Fang, 2008; 2012; Halliday, 1989; Nagy y Townsend, 2012) tiene las siguientes características:

- Palabras morfológicamente complejas.

- Mayor recurso a sustantivos, menor cantidad de adjetivos y verbos.

- Palabras de origen latino o griego.

- Metáfora gramatical, sobre todo nominalizaciones.

- Densidad informativa.

- Carácter abstracto de la información.

Estas características de los textos académicos están relacionadas, es decir son interdependientes. Las palabras morfológicamente complejas, los sustantivos abstractos y derivados, las nominalizaciones y las palabras de origen latino o griego contribuyen a dar una mayor densidad a 
la información al presentar aspectos técnicos y abstractos en los textos. En general, el vocabulario académico reposa sobre construcciones pluri-morfémicas y sobre construcciones con derivados. En efecto, la mayoría de las palabras del vocabulario académico tiene más de un morfema: las palabras pluri-morfémicas constituyen más del 60\% del vocabulario de un alumno antes de los 14 años (Rey-Debove, 1984). En muchos casos, el sentido de las palabras académicas es construido y se puede predecir a partir de su estructura morfológica (Corbin, 1991), aunque su predicción dependa del conocimiento del lector y, por ello, a veces esa misma estructura deviene opaca (Booij, 2015). Además, según Coxhead (2000), más del 90\% del vocabulario académico son palabras complejas que vienen del latín o del griego, muchas de ellas con prefijos y sufijos.

La característica de presentar metáforas gramaticales se usa para referirse a los fenómenos sociales y naturales, y consisten en el uso de sustantivos que derivan de verbos o de adjetivos, denominados «nominalizaciones», y que sustituyen a una clase de palabras o una estructura gra-matical (Chamorro, Barleta y Mizuno, 2013; Fang, 2008; Halliday, 1989). La metáfora gramatical con nominalización hace referencia a los procesos y a las propiedades en forma de sustantivos. Para comprenderlos es necesario reconstruir su estructura argumental, por ejemplo, del verbo del que derivan. Por tanto, aunque con forma de sustantivo, una nominalización no se refiere a obje-tos sino a procesos; y es una metáfora que implica una paráfrasis de las cláusulas oracionales a las que sustituye (Peris, 2012). Por ejemplo, en el extracto citado son nominalizaciones las siguientes palabras: precipitaciones (de precipitar), evaporación (de evaporar), temperatura (nominalización del latín, temperare con el sufijo -ura, acción, resultado).

La densidad informativa se consigue con la concentración de muchas palabras de con-tenido (sustantivos, verbos, adjetivos); y una manera de medirla es calcular el número medio de palabras de contenido por oración (Fang, 2008). El carácter abstracto de la información se da por el uso no sólo de palabras de contenido sino por el vocabulario técnico, por las nominalizaciones con sustantivos derivados que contribuyen a dificultar la comprensión por la dependencia de los enlaces con otras palabras y frases.

\subsection{Exigencias de comprensión}

En cuanto a los alumnos, cada una de las anteriores características del texto presenta exigencias de conocimientos previos. Es decir, para que los alumnos adquieran conocimientos a partir de la lectura del texto, necesitan poseer conocimientos del mundo para comprender el discurso del texto (Van Dijk, 2010:168). Informes recientes (Biancarosa y Snow, 2006; Fang y Schleppegrell, 2008; Moje, 2008) sugieren que más del 70\% de los estudiantes en los grados a partir de $4^{\circ}$ curso experimentan dificultades al leer y escribir textos en áreas de contenido académico. Por ejemplo, las palabras técnicas necesitan un cierto nivel de análisis de su estructura como una estrategia de aprendizaje. En el extracto citado, la palabra atmósfera viene del griego (atmós = vapor y sphaira = esfera), la palabra temperatura del latín (= temperare), así como las palabras precipitaciones y meteorología. Los sustantivos o adjetivos poco frecuentes y de tipo técnico son a menudo difíciles de comprender y requieren una exposición repetida en los textos impresos (Cunningham y Stanovich, 1991; Nagy, Herman y Anderson, 1985; Nippold y Sun, 2008). Por ello, en el anterior texto, como en muchos otros, se presentan en definiciones entrelazadas: cada definición se utiliza para 
definir un concepto que es condición para entender el concepto siguiente.

Del mismo modo que las definiciones, las nominalizaciones, los derivados y los términos técnicos denotan aspectos abstractos, intangibles, con complejidad semántica (Nippold y Sun, 2008). Todas las características de los textos de contenido disciplinar presentan requisitos de análisis, requisitos que a su vez implican una competencia metalingüística.

\subsection{Condiciones de los alumnos}

Hemos mencionado de manera general «alumnos de $4^{\circ}$ curso», pero numerosos estudios señalan que en el conocimiento del vocabulario hay grandes diferencias entre los niños, incluso en un solo grupo clase. Varios factores pueden estar implicados en estas diferencias: la brecha entre las actividades de producción y de comprensión, la distancia entre el rendimiento oral y el escrito, las características de la lengua, las oportunidades sociales y culturales o la aptitud de cada niño influyen para que algunos niños adquieran más significados que otros niños. En particular, el nivel socioeconómico y cultural que crea desigualdad, también en cuanto al lenguaje académico y el vocabulario. Esa desigualdad es tanto cuantitativa: los ricos tienen más palabras que los pobres, como cualitativa: hay palabras de ricos, palabras de pobres (según el título del libro de J.-L. Fournier, 2006). Fournier presenta como supuesto de partida que ricos y pobres viven en mundos diferentes, no sólo en términos materiales, sino también en relación a la mentalidad, en particular en relación al vocabulario que no es neutral respecto a los contextos de desarrollo social (Paveau, 2006). Por ello, la distancia entre lenguaje escolar y familiar puede ser grande en algunos colectivos.

En el tema de la desigualdad en el vocabulario asociada al nivel socioeconómico, el estudio más frecuentemente citado es el de Hart y Risley (1999). Estos autores estimaron en Estados Unidos que la diferencia según los grupos socioeconómicos era muy grande: un niño de una familia profesional estaría expuesto a una experiencia acumulada de aproximadamente 42 millones de palabras, en comparación con los 13 millones de palabras de un niño de una familia de bajo nivel socioeconómico. Esta diferencia crea una desigualdad lingüística acumulada que puede influir en la comprensión lectora y en el desempeño académico (Biemiller y Boote, 2006).

En resumen, hay una influencia recíproca entre conocimiento del vocabulario y comprensión de lo escrito. Los estudios muestran que en el desarrollo del lenguaje existe una relación entre una «masa crítica» de conocimiento lexical y la emergencia de la gramática (Bates y Goodman, 1997:11). Otros estudios sugieren que hay una relación entre la comprensión de lo que se lee, el éxito escolar, y la adquisición de vocabulario, así como la sintaxis y el conocimiento académico (Beck y McKeown, 2007, Cunningham y Stanovich, 1997; Snow y Uccelli, 2009). Teniendo en cuenta estas evidencias sobre la importancia del vocabulario, consideramos que tiene considerable importancia en el desarrollo disciplinar.

La doble influencia se explica porque, en primer lugar, la falta de vocabulario influye sobre la capacidad de reconocimiento de las palabras gráficas y de conocer su significado -y por lo tanto de llegar a una cierta automatización y comprensión en la lectura. En segundo lugar, la dificultad de estos lectores, el desconocimiento de muchas palabras y la poca comprensión del texto disminuye el entusiasmo por la lectura. La relación entre conocimiento de vocabulario y lectura sufre lo que se ha denominado como el «efecto Mateo» (Stanovitch, 1986): cuanto mayor es la dificultad, 
menor es la lectura. Esta relación recíproca se da no solo en relación a la lectura sino también en la producción de textos (García y González, 2006).

\subsection{Condiciones de la enseñanza}

Pero ¿es el vocabulario objeto específico de enseñanza? En las Orientaciones curriculares en el ámbito escolar donde realizamos nuestro estudio ${ }^{1}$, el vocabulario no suele ser objeto de enseñanza sistemática; y la mayoría de las prácticas pedagógicas de la escuela Primaria no suelen incorporarlo. Se espera que su aprendizaje sea el resultado de un enfoque muy general, como un complemento derivado de la lectura. Por otra parte, muchas veces los profesores no disponen de instrumentos eficaces para su enseñanza. Por ejemplo, uno de los problemas entre los profesores y en los manuales escolares es la ausencia de una conceptualización de la unidad lexical en la enseñanza del vocabulario, unidad a la que denominamos generalmente palabra. Esta denominación intuitiva puede ser útil en la escuela, e incluso en el diálogo con los alumnos, a condición de que no enmascare diferenciaciones importantes, como la diferencia entre palabra como unidad gráfica y palabra como unidad oral. Además, ¿qué cuenta como una palabra? Podemos contar cada forma de ocurrencia (por ejemplo, perro y perros), en ese caso contaríamos lo que se denomina ocurrencias, o solo contar una forma sin considerar las variaciones morfológicas, es decir contar los tipos (diferencia type/tokens, en inglés). Por otra parte, solemos incluir bajo la denominación «palabra» entidades de dos clases diferentes: entidades de la clase abierta o lexical y de la clase cerrada o gramatical. Pero, la palabra (sea en oral o en escrito) no siempre es una unidad simple, sino que muchas veces es derivada y construida por elementos morfológicos, o sea una unidad pluri-morfémica. Así también, no hay que considerar solo las unidades aisladas, sino también las construcciones pluri-lexicales. (Colé, 2011; Nation, 2001; Petit, 2000). Por ello, es necesaria una conceptualización de las unidades del vocabulario.

En los manuales, en las orientaciones didácticas, y en las aulas donde todavía se practica, se suele estimular ejercicios de «familia de palabras» como único tipo de relación entre las palabras. Estos ejercicios consisten básicamente en agrupar las palabras que comparten raíz histórica (etimológica). Pero, desde el punto de vista didáctico, es también conveniente abarcar relaciones semánticas más amplias. Cuando las unidades léxicas forman parte de un mismo dominio y tienen el mismo identificador conceptual o temático, se dice que forman un «campo semántico». Por ejemplo, lluvia, nieve, granizo, niebla y tormenta tienen el mismo componente identificador de «fenómenos atmosféricos», del que podemos extraer una información común de ese campo (Grossmann, 2011).

En este estudio, cuando hablamos de vocabulario no solamente nos referimos a la cantidad de palabras que los niños han acumulado a una cierta edad, también incluimos las relaciones entre palabras en dimensiones sintagmáticas y paradigmáticas de la lengua. Es decir, no solo es cuestión del incremento del vocabulario, sino fundamentalmente de las relaciones, porque cada palabra puede ser considerada como el centro de una red de relaciones de tipo semántica, sintáctica, morfológica, ortográfica.

1 En Barcelona, según las Competències bàsiques de l’àmbit lingüístic (2015) presentadas por el Departament d’Ensenyament de la Generalitat de Catalunya. 
Dada la distancia y las diferencias entre el vocabulario propuesto en los libros de textos, orientaciones, etc. y el que dominan los alumnos, hay consenso entre los estudios sobre que el conocimiento de vocabulario es una llave de acceso al conocimiento en todas las disciplinas. Por ello, muchos autores han propuesto enseñar vocabulario junto con la lectura y la escritura (David, 2003; Calaque, 2004; Grossmann, 2011) o realizar intervenciones tempranas para enfocar mejoras en el vocabulario de los niños (Neuman y Dwyer, 2011). Nuestra experiencia de intervención sigue esta línea, teniendo en cuenta que se trata de un vocabulario técnico, abstracto, multi-morfémico, pluri-lexical, con un origen etimológico desconocido para los alumnos.

\section{Propuesta del presente estudio}

En el presente artículo presentamos una propuesta para abordar la enseñanza-aprendizaje-comprensión a partir de leer textos, focalizando sobre el aprendizaje del vocabulario de los textos académicos y proponiendo actividades para promover este aprendizaje. Esta propuesta se basa en una experiencia realizada en una escuela pública de Barcelona .

Dadas las características de los textos que los alumnos deben comprender y las dificultades de aprendizaje y enseñanza antes señaladas, decidimos proponer una situación de formación a una profesora de $4^{\circ}$ curso de Primaria con un diseño de actividades que focalice especialmente sobre el vocabulario con el objetivo de mejorar la comprensión de textos. Nos basábamos en las evidencias de los estudios para acercar los resultados de la investigación a la práctica educativa, en un experimento formativo basado en diseñar actividades en el aula (Reinking y Bradley, 2004).

Por ello, diseñamos la intervención centrándonos en tres objetivos de aprendizaje: (i) en la libre selección y organización de las palabras que hacen los alumnos cuando se les propone buscar las que están relacionadas con el tema de «fenómenos atmosféricos» de Ciencias naturales y en la comparación de estas palabras entre sí para encontrar elementos en común. Puesto que la selección de palabras relacionadas con el tema de estudio era libre, nos interesaba saber cuáles eran los criterios de selección, si eran palabras familiares o palabras académicamente sofisticadas las que escogían los alumnos. (ii) Como segundo objetivo nos interesaba comprender cuáles eran los procedimientos de organización del vocabulario seleccionado y averiguar los criterios de agrupación que usaban. (iii) En tercer lugar, queríamos analizar las respuestas a la propuesta de comparar entre sí las palabras para identificar los elementos comunes de misma forma y de sentido (o morfemas) en la composición de las palabras.

En cuanto a las actividades, en la intervención propusimos una serie encadenada y contextualizada dónde las tareas se sucedían a lo largo del trimestre. En la práctica, esta serie comenzaba por la lectura en voz alta de un texto sobre los «fenómenos atmosféricos» por parte de la profesora, lectura acompañada por su visualización digital y por comentarios sobre el contenido textual. ¿Qué aporta la lectura en voz alta y la visualización en estas edades? Según varios autores (Middleton, 2005; Svendro, 1997), esta situación crea un ambiente que, como en una obra de teatro con participación de la audiencia aporta ser consciente de la co-presencia en el grupo. Las personas llegan a compartir conocimientos y emociones en esta situación conjunta. Además de la voz al texto, la profesora completaba la presentación con comentarios e interpretaciones para ayudar a entender su contenido y facilitar la comprensión. 
Luego se realizaban las actividades sobre el vocabulario relacionado con el tema del texto y con su organización semántica. Las tareas sobre el vocabulario consistían en buscar, seleccionar, hacer listas y organizar las palabras de las listas. La primera actividad era seleccionar las palabras del texto. Desde una perspectiva cognitiva amplia, se sabe que el léxico es fundamental para abordar el tema del texto, mantener la coherencia, organizar sus partes y dar cuenta de la estructura del mismo (Bernárdez, 2010).

La segunda actividad consistía en ampliar la lista de las palabras del texto con otras relacionadas con el tema a través de la consulta a diccionarios o a la web, el eje en este caso residía en la lista. El uso de la lista, una tecnología léxica de larga historia (Sepúlveda y Teberosky, 2008), sirve para el seguimiento y la identificación de las unidades léxicas, y tiene una función cognitiva de memoria externa y de organización del mundo y de la experiencia. Por lo tanto, es considerada como una herramienta que ayuda en el aprendizaje, en la memoria y como modo de categorización. Hay evidencias empíricas de que las listas de palabras sirven como «invitaciones para formar categorías» (Waxman y Markow, 1995). Por ello, propusimos a los alumnos una lista como primera actividad; lista que debían tener una forma material y servir como herramienta de búsqueda léxica para llegar a un inventario de los componentes identificadores del tema «fenómenos atmosféricos».

Las decisiones pedagógicas al proponer hacer una lista, según Nation (2015), son explicitar la razón de para qué se hace, reflexionar sobre el tipo de texto que es usado como fuente de las palabras (porque la naturaleza de la fuente determina el tipo de palabra) y observar cuál es la unidad de la lista que determina el tipo de palabra, puesto que incluso un cambio en la forma resulta en un tipo diferente (por ejemplo, no es lo mismo libro que libros, incluso, a veces la tipografía influye: libro no es lo mismo que LIBRO, o todo cambio en la flexión o derivación).

Estas decisiones pedagógicas también responden a una descripción del aprendizaje, almacenamiento y procesamiento lexical, que como sostiene Bybee (1998:423) no es solo declarativo, sino básicamente procedimental, es decir, es un conocimiento que está muy afectado por la repetición y el uso.

Una vez construidas las listas, se propuso que se orientara a los alumnos para que procedieran a comparar las palabras, con el fin de descubrir cómo estaban formadas y de encontrar el elemento común y los elementos morfológicos de composición y derivación. Como hemos comentado respecto al contenido temático del estudio, la comparación sirve porque muchas palabras son morfológicamente complejas y de origen latino o griego. Para ello, seguimos las propuestas de Bybee (1998), Rey-Debove (2004) y Lehmann (2011) de estimular un análisis por comparaciones de elementos comunes entre las palabras que comparten un mismo origen.

Respecto a la comparación como procedimiento se justifica porque la estructura interna de la palabra deriva de conjuntos de conexiones hechas con otras palabras que comparten partes relacionadas (morfemas). La primera etapa de la comparación era para descubrir las raíces que las palabras, que no tienen representación separada, sólo existen como relaciones de similitud entre palabras (Bybee, 1998:422).

En una segunda etapa propusimos la comparación entre las palabras para descubrir y analizar los elementos afijos (prefijos y sufijos) y buscar sus definiciones. Finalmente, la serie de actividades acababa con la búsqueda de definiciones de algunas palabras de la lista. A partir de 
etiquetas con los elementos comunes, los alumnos tenían que escribir las definiciones. Luego la profesora separó ambas etiquetas pidiendo que, a partir de la lectura de la definición, encontraran el elemento definido. Se organizó la tarea como un juego en el que tenían que hacer corresponder el término definido y la definición. Finalmente, se propuso un análisis básico de tipo gramatical para categorizar las palabras de las listas en términos de nombres, verbos y adjetivos.

\section{Metodología}

La intervención realizada en el presente estudio es del tipo denominado «experimento formativo», que consiste en planear las actividades de clase con el objetivo de aumentar el aprendizaje de pro-fesores y alumnos, como un logro de un proceso de enseñanza y aprendizaje (Reinking y Watkins, 2000; Reinking y Bradley, 2004). La intervención formativa que presentamos aquí involucró a los alumnos y su profesora de cuarto curso de Primaria. En concreto, pretendíamos aumentar la cantidad y la diversidad de la lectura y la comprensión de textos del tema estudiado.

Estos experimentos de formación no suelen requerir grupos control como en los experi-mentos convencionales, porque se supone que habrá cambios y se busca más la validez y coheren-cia interna que la comparación externa (Reinking y Watkins, 2000). Del mismo modo, los datos cuantitativos no necesariamente se usan para establecer relaciones causales, sino para apoyar o refutar las inferencias sobre los vínculos entre ciertos factores o acontecimientos, o la «credibili-dad explicativa» (como la denominan Reinking y Watkins, 2000:398, citando a Krathwohl, 1993). En cuanto a la transferencia de los resultados de este estudio, no se pueden descuidar factores tales como el contexto y el tiempo de una experiencia formativa. El contexto en el que trabajamos implica a toda la escuela y profesores del nivel Infantil y Primaria, desde hace 8 años. Los alum-nos que participaron en el presente estudio ya habían seguido situaciones similares en sus cursos anteriores. Eso no excluye, evidentemente, la posibilidad de generalización, pero si limita las com-paraciones directas.

El desarrollo de la intervención en las clases fue observado durante 12 semanas, con una frecuencia de 2 días consecutivos a la semana para dar continuidad al seguimiento. Las actividades programadas tenían una duración de una hora y media; en total fueron 36 horas de observación directa grabadas en vídeo durante la intervención. El objetivo de nuestras observaciones y gra-baciones era el registro del discurso y la actividad de la profesora, así como de las interacciones entre profesora y alumnado. Queríamos saber cómo la profesora introducía el tema, cómo y con qué consigna distribuía las tareas entre los alumnos y cuáles eran sus orientaciones. El método utilizado fue semejante a un análisis etnográfico, es decir registrar lo que se hizo y dijo en clase, sin categorías predefinidas tal como propone N. Mercer (1995:68). Nuestro interés estaba en registrar el desarrollo de las actividades de clase. La transcripción de lo que se había grabado en vídeo servía hacer un análisis del desarrollo de las actividades, así como un análisis de las producciones escritas hechas por los alumnos de forma individual o en los grupos.

\subsection{Participantes}

Este estudio se realizó en una escuela primaria pública de Barcelona. La profesora participó con compromiso con el proyecto formativo, estableciendo una relación colaborativa con las investiga- 
doras; y los niños mostraron interés y entusiasmo en las actividades. Participaron 24 niños de $4^{\circ}$ curso de primaria (entre 9 y 10 años) que se organizaron en 6 grupos de trabajo. De esta agrupación se debe destacar, según su profesora, que los grupos estaban formados por, al menos, uno o dos alumnos con un nivel medio o alto de rendimiento.

\subsection{Materiales y consignas}

Los materiales didácticos utilizados durante la intervención corresponden a recursos impresos como el «texto-fuente» preparado por la profesora, enciclopedias, libros de consulta y recursos digitales variados en portátiles y tabletas, de donde los alumnos extrajeron información de internet. Las consignas para las tareas por parte de la profesora fueron: (a) «a partir de los textos que hemos trabajado y de la información que han traído de casa, hacer una lista de palabras relacionadas con el tema de la meteorología. Lo haréis en estas hojas de forma vertical, se debe poner el título, numerar las palabras y hacer una letra que se entienda bien; iremos ampliando esta lista a medida que vamos trabajando el tema, utilizando las tabletas y portátiles». Es decir, se trataba de una tarea de selección en los textos y de organización de las palabras encontradas. (b) Al finalizar esta tarea, se solicitó una lectura de la lista y se sugirió a los alumnos que compararan las palabras, «para encontrar lo que tienen de semejante, lo que tienen en común», preguntando a cada grupo «¿cuál es el elemento común?». Se trataba de una tarea de comparación para establecer las analogías entre las palabras. Una vez finalizada la serie de tareas, (c) se pidió un análisis, para «encontrar las partes comunes». Luego, (d) se promovió una tarea de definición de palabras y morfemas; y finalmente (e) se orientó a los alumnos para hacer una categorización gramatical con la siguiente consigna: «aunque no tengan elementos en común, ¿en qué se pueden parecer palabras como nadar, airear, gotear, correr?».

\subsection{Análisis de las producciones de los alumnos}

Como hemos comentado los grupos presentaban diferente nivel de rendimiento. Para establecer los análisis de las producciones utilizamos la estrategia de comparar las listas con vocabulario más pequeño respecto a las listas con mayor cantidad de palabras. Se trata de una estrategia su-gerida por Biemiller (2012; Biemiller y Boote, 2006) que sostiene que, en gran medida, los niños aprenden principalmente las mismas palabras a ritmos diferentes. Es decir, los niños aprenden las palabras en un orden predecible. En base a esta comparación se analizaron las listas y se estableció cuáles eran las palabras compartidas entre los grupos y cuáles eran exclusivas de un grupo. Se comparó el nivel de dificultad en función de las palabras compartidas o no compartidas por los grupos, del modo siguiente (según Biemiller, 2012:198):

- Fáciles: palabras incluidas por $80 \%$ o más de los niños.

- De dificultad media: palabras incluidas por 40-79\% de los niños.

- Con dificultad: palabras incluidas por menos de $40 \%$ de los niños.

\section{Resultados de las actividades de clase}

Los alumnos agrupados en 6 grupos llegaron a hacer listas de entre 60 y 117 palabras. Analizando sus producciones observamos, en primer lugar, que en carteles la forma material de las palabras en 
la lista era vertical. Las producciones muestran que la razón de seleccionar las palabras para la lista fue su relación con el tema, y cuando se encontraban con una palabra desconocida, buscaban en el diccionario su significado y decidían si incorporarla a la lista. Las fuentes consultadas para buscar las palabras relacionadas fueron textos escritos en libros, diccionarios en papel y enciclopedias en internet (que funcionaron como textos fuente de ampliación del vocabulario). Respecto a la unidad de la lista los niños seleccionaron lemas de la palabra principal, sin flexiones ni derivaciones. Todas las palabras eran pertinentes desde el punto de vista semántico.

\subsection{La fuente para la selección de las palabras de la lista}

En respuesta a la consigna de hacer listas de palabras relacionadas con el tema de la meteorología, los niños seleccionaron primero las palabras del texto-fuente (que funcionó como introducción al tema, seleccionado de una enciclopedia), como puede verse en el conteo siguiente:

- Grupo 1: 117 palabras, 36 coincidente con T-F (30,76\%)

- Grupo 2: 94 palabras, 27 de coincidentes con T-F (28,72\%)

- Grupo 3: 82 palabras, 35 coincidente con T-F (42,68\%)

- Grupo 4: 58 palabras, 25 coincidente con T-F $(43,10 \%)$

- Grupo 5: 57 palabras, 18 coincidente con T-F $(31,57 \%)$

- Grupo 6: 60 palabras, 12 coincidentes con T-F (38,70\%)

La cantidad de palabras seleccionadas difiere mucho entre los grupos: los más productivos (grupos 1 y 2) buscaron en otros textos, los menos productivos agotaron más el texto-fuente.

\subsection{La selección de palabras o cómo los niños interpretaron la consigna}

Los niños seleccionaron no solo palabras diferentes sino también de la misma categoría gramatical: nombres y frases nominales. La selección de las palabras de todos los niños fue sustantiva. Es interesante señalar que la profesora había indicado «hacer una lista de palabras»; y los niños interpretaron como «hacer una lista de nombres» (esta interpretación coincide con los resultados de otras investigaciones, Ferreiro y Vernon, 1992; Ferreiro, 2002). En ella puede estar implícita la dificultad debida al metalenguaje de la consigna y la falta de distinción conceptual entre "palabra» $\mathrm{y}$ «nombre». La excepción en estas listas de nombres fueron algunas construcciones de frases nominales que incluían adjetivos, como cambio climático, viento suave, lluvia intensa, etc.

Pero la anterior interpretación puede coincidir con otra: la actividad de hacer listas llevó a los niños a una organización conceptual del dominio de conocimiento, en particular a su categorización. En efecto, sabemos que hay una relación estrecha entre lenguaje y conceptos, como lo han demostrado varios estudios antes citados.

\subsection{La organización temática: categorial y en series de las listas}

En cumplimiento de la tarea, los alumnos seccionaron palabras que tenían relación temática con el tema estudiado. Pero en los grupos más productivos, las palabras se seleccionaron y organizaron también con relaciones taxonómicas o con relaciones en series. (algunas de las relaciones se presentan en la Tabla 1). 


\begin{tabular}{|l|l|}
\hline Serie de estaciones del año & verano, otoño, invierno, primavera \\
\hline Series de intensidad del viento & calma, brisa, bastante viento, viento fuerte \\
\hline Clases de viento & viento, viento fuerte, viento suave, viento variable, ventisca \\
\hline Clases de catástrofes naturales & Inundación, tornado, tsunami, aludes, huracán \\
\hline Clases de precipitaciones & Gotas, aguacero, chaparrón, rociado, granizo, piedra, nieve, nevada \\
\hline Localización geográfica & $\begin{array}{l}\text { Ecuador, Polo norte, Polo sur, Trópico de cáncer, Trópico de Capricornio, } \\
\text { Círculo polar }\end{array}$ \\
\hline Clases de vientos & Tramuntana, Gregal, Llevant, Xaloc, Migjorn, Garbí, Mestral \\
\hline Clases de instrumentos & Pluviómetro, anemómetro, termómetro, barómetro \\
\hline Clases de estados del cielo & Nublado, sereno, cúmulos, soleado, cubierto, claro \\
\hline
\end{tabular}

Tabla 1. Series temáticas.

\subsection{Comparación entre las listas: palabras compartidas}

Como hemos dicho, los listados presentaron un total de entre 60 y 117 palabras, con algunas palabras compartidas y otras diferentes entre los grupos de clase; el rango de palabras compartidas fue de entre 2 y 6 veces (en los grupos de niños) de palabras citadas en las listas.

\begin{tabular}{|l|l|}
\hline $\begin{array}{l}6 \text { veces } \\
(=13)\end{array}$ & $\begin{array}{l}\text { Calamarsa, Fred, Migjorn, Plugim, Pluja, Pluviòmetre, Núvols, Temps, Sol, Tornado, Tramuntana, } \\
\text { Neu, Clima }\end{array}$ \\
\hline $\begin{array}{l}5 \text { veces } \\
(=14)\end{array}$ & $\begin{array}{l}\text { Anemòmetre, Brisa, Calor, Ennuvolat, Llevant, Meteorologia, Ponent, Precipitacions, Sequera, } \\
\text { Temperatura, Tempesta, Trons, Tsunami, Vent }\end{array}$ \\
\hline $\begin{array}{l}4 \text { veces } \\
(=11)\end{array}$ & Assolellat, Boira, Cel serè, Cel, Gel, Hivern, Humitat, Llamps, Mestral, Primavera, Tardor \\
\hline $\begin{array}{l}3 \text { veces } \\
(=17)\end{array}$ & $\begin{array}{l}\text { Atmosfera, Congelat, Energia, Estiu, Garbí, Gelada, Huracà, Llampec, Mar, Mar alta, Meteosat, } \\
\text { Penell, Rosa dels vents, Solades, Termòmetre, Vapor, Xaloc }\end{array}$ \\
\hline $\begin{array}{l}2 \text { veces } \\
(=39)\end{array}$ & $\begin{array}{l}\text { Aigua, Aire, Allaus, Anticicló, Arc de Sant Martí, Baròmetre, Borrasca, Calma, Cicle de I'aigua, } \\
\text { Cicló, Cirrus, Climatitzat, Climograma, Cúmulus, Depressió, Detector de Ilamps, Equador, Gasos, } \\
\text { Graus, Gregal, Huracà, Llampec, Marea, Marea baixa, Meteorit, Nevada, Núvol, Panell, Pedrega- } \\
\text { da, Pressió atmosfèrica, Rolades, Ruixat, Serè, Solar, Tapat, Terratrèmol, Ventada, Ventijol, Ventisca }\end{array}$ \\
\hline
\end{tabular}

Tabla 2. Palabras compartidas.

\subsection{Palabras no compartidas}

Entre las palabras no repetidas en el grupo que obtuvo mayor productividad debemos incluir las más sofisticadas y multi-morfémicas, las técnicas y las construcciones pluri-lexicales. Por ejemplo, presión atmosférica, temperatura atmosférica; barómetro, anemómetro, pluviómetro, estratus, Fahrenheit; zonas de bajas presiones, rosa de los vientos, cambio climático, capa de ozono, etc. En cambio, en los grupos menos productivos las palabras eran más frecuentes.

\subsection{Nivel de dificultad de las palabras seleccionadas}

Hemos seguido el criterio de Biemiller $(2009,2012)$ para establecer el nivel de dificultad: las más fáciles eran las palabras incluidas por $80 \%$ o más de los niños, de un nivel medio eran las incluidas por 40-79\% de los niños y las palabras más difíciles eran las incluidas por menos de $40 \%$ de los niños. 
En nuestro corpus, de las 94 palabras compartidas por los grupos, resultaron:

- 39 palabras fueron incluidas por un $41,48 \%$, pueden considerarse palabras fáciles,

- 42 palabras fueron incluidas por un $44,68 \%$, pueden considerarse de un nivel intermedio; $y$

- 13 palabras fueron exclusivas de un 13,82\% del total, pueden considerarse más difíciles.

Como podemos ver aproximadamente la mitad de la clase se encuentra en el nivel intermedio de dificultad, habiendo seleccionado palabras frecuentes, y solo una minoría fue capaz de seleccionar palabras exclusivas, poco frecuentes y más difíciles.

\subsection{La comparación entre las palabras a través de elementos en común}

A partir de las listas escritas, los alumnos tuvieron que identificar y subrayar el elemento común de la raíz de las palabras. A partir de este criterio, tenían que agrupar las palabras con un elemento común (por ejemplo, mar, marea; sol, solar, etc.). La profesora estimulaba las comparaciones y hacía observaciones sobre la actividad y las producciones de los alumnos. En la tarea de comparación para la extracción de la raíz, los niños debían encontrar otra palabra más pequeña dentro de la palabra presentada. Se trataba de aprender a comparar las palabras para descubrir la existencia de familias de palabras unidas por un elemento común (ver Tabla 2).

Luego de la comparación, los alumnos recibieron la orientación de la profesora para descubrir cómo se descomponía la palabra. A partir de esta actividad, se les estimuló a buscar el significado de los morfemas, sean raíces, prefijos o sufijos. Las preguntas que nos hicimos desde la intervención eran: ¿podrían los alumnos identificar los elementos comunes?, ¿podrían hacer un análisis morfológico?, ¿llegarían a identificar la raíz de las palabras, a diferenciar prefijos y sufijos? El resultado de la comparación se reflejó en una sola tabla, de la forma siguiente:

\begin{tabular}{|l|l|l|l|l|l|l|}
\hline Clima & Climàtic & Climograma & Climes & Climatològic & Climatologia & Aclimatitzat \\
\hline Termòmetre & Baròmetre & Higròmetre & Anemòmetre & Pluviòmetre & Udòmetre & \\
\hline Vents & Ventijol & Vent & Ventisca & Ventada & & \\
\hline Altocúmulus & Cúmulus & Cumulonimbus & Cirrocúmulus & & & \\
\hline Núvols & Nuvolat & Ennuvolat & Núvol & Nuvolositats & & \\
\hline Llamps & Parallamps & Llampecs & & & & \\
\hline Meteoròlegs & Meteorit & Meteoròleg & Meteorologia & Meteorològiques & Meteosat & Meteorològics \\
\hline Assolellat & Sol & Solar & Solades & & & \\
\hline Pluja & Pluviòmetre & Plugim & Plujós & & & \\
\hline Gelat & Congelat & Gelada & Gel & & & \\
\hline Cicló & Anticicló & & & & & \\
\hline Atmosfera & Atmosfèric & & & & & \\
\hline Neu & Nevada & & & & & \\
\hline Mar & Marea & & & & & \\
\hline
\end{tabular}

Tabla 3. Palabras con elemento común.

La respuesta de los alumnos fue positiva: hicieron la actividad de comparar, manipular y descubrir la composición morfológica y el acceso a las partes (raíces, prefijos y sufijos), subrayaron los elementos comunes, agruparon las palabras y comenzaron a buscar significados. 


\subsection{Sufijos y prefijos}

A partir de esta comparación, además de la raíz, se orientó a los alumnos a analizar los sufijos y prefijos comunes (del corpus del campo semántico fenómenos atmosféricos). Los afijos seleccionados representaban una alta frecuencia entre los términos académicos. Los alumnos buscaron el significado de las palabras nuevas aplicando la definición también a los afijos. Luego buscaron ejemplos de palabras nuevas que los incluyeran. Esta búsqueda fue libre y por tanto se incluían palabras de un campo semántico diferente al de fenómenos atmosféricos, luego hicieron una tabla con las palabras encontradas (ver Tablas 4 y 5 . Hemos hecho la traducción de la definición, manteniendo los ejemplos en la lengua original).

\begin{tabular}{|l|l|l|}
\hline \multicolumn{1}{|c|}{ Sufijos } & \multicolumn{1}{|c|}{ Definición } & \multicolumn{1}{c|}{ Ejemplos con afijos en común (en catalán) } \\
\hline Itat & $\begin{array}{l}\text { Sufijo de formación de nombres femeninos y } \\
\text { que significa calidad de: dignidad, movilidad, } \\
\text { profundidad }\end{array}$ & profunditat, dignitat, velocitat, nuvolositat \\
\hline Ment & Efecto: manera de hacer las cosas & ràpidament, ensenyament, pensament, reglament \\
\hline Ada & Conjunto colectivo, que hay muchas & olivada, ventada, nevada, costellada \\
\hline Ejar & Frecuencia, que se hace muchas veces & gotejar, airejar, vesprejar, aletejar \\
\hline Ós & Significa abundancia & plujós, verdós \\
\hline Logia & Significa tratado, teoría, ciencia & climatologia, meteorologia, filologia, zoologia \\
\hline Leg/loga & $\begin{array}{l}\text { Leg significa la formación de nombres. Los que } \\
\text { lo estudian }\end{array}$ & meteoròleg, geòleg \\
\hline At/ada & $\begin{array}{l}\text { Adjetivo que indica una calidad de un nombre } \\
\text { o de otro adjetivo }\end{array}$ & $\begin{array}{l}\text { ennuvolat, gelada, congelat, pigada, assolellat, } \\
\text { descongelat, gelat, nuvolat, nuvolositats, nevat }\end{array}$ \\
\hline Ista & $\begin{array}{l}\text { Sufijo que entra en la formación de nombres y } \\
\text { significa oficio }\end{array}$ & taxista, maquinista \\
\hline Dor & $\begin{array}{l}\text { Sufijo que entra en la formación de nombres de } \\
\text { adjetivos y nombres y significa el sitio. }\end{array}$ & congelador, menjador \\
\hline Ar & Hacer una acción. & Cantar, ballar, nedar \\
\hline Dor/a & Persona que hace la acción u oficio. & Entrenador, pescador \\
\hline
\end{tabular}

Tabla 4. Sufijos, definición y ejemplos.

\begin{tabular}{|l|l|l|}
\hline Prefijos & \multicolumn{1}{|c|}{ Definición } & \multicolumn{1}{c|}{ Ejemplos con afijos en común (en catalán) } \\
\hline Avant & En el interior o delante & avantpassat, avantbraç \\
\hline Ante & Anterior & antepenúltim, anteposar \\
\hline Micro & Pequeño & microones, microcircuit \\
\hline Amfi & Quiere decir de 2 maneras & amfibi, amfiteatre \\
\hline Super & Más allá, en abundancia & superfície, supermercat \\
\hline Multi & Muchos & multicolor, multicultura, multimilionari \\
\hline Sota & Debajo & sotabosc, sotavent \\
\hline Auto & Uno mismo & autobús, autocar, autoestima \\
\hline Contra & Nociones de oposición, complementariedad & contradir, contraatacar \\
\hline Para & Cerca de & parallamp, paraigua \\
\hline Anti & Opuesto & antimosquits, antibiòtic \\
\hline Ex & Hacia afuera, anterior & excampiona, exnovio \\
\hline
\end{tabular}

Tabla 5. Prefijos, definición y ejemplos. 
Como se puede observar, los alumnos llegaron a separar prefijos y sufijos y a definirlos. Los ejemplos fueron seleccionados por los niños, algunos relacionados con el tema, otros en función de la definición de los afijos.

\subsection{Comprender y definir}

Comprender el vocabulario y tener una explicación del significado era otra actividad con las palabras encontradas. Es decir, dar una respuesta a "qué quiere decir», respuesta que se podía realizar de diversas formas: a través de una definición evidentemente, pero también por una paráfrasis, con ejemplos, con ilustraciones, proponiendo antónimos o sinónimos, etc., en los términos usados por los alumnos. Luego tenían que escribir en carteles las definiciones, ejemplos, comentarios, etc. de las palabras académicas, establecer relaciones entre la definición y el término definido para garantizar la memorización del mismo. Esta actividad se repetió en varias ocasiones para estimular la memoria. Nuestras preguntas estaban en relación a: ¿cómo harían las definiciones de términos técnicos?, ¿podrían establecer las relaciones entre definición y término definido?

Con la ayuda de la profesora que orientó a los alumnos a buscar las definiciones de las palabras raíces y de los afijos. Algunas de estas definiciones son copia del diccionario o paráfrasis de la definición y/o de la explicación oral de la profesora. El resultado fue de 18 definiciones (ver la siguiente Tabla 6).

\begin{tabular}{|l|l|}
\hline Viento & Del latín ventus: viento movimiento natural, generalmente horizontal, de masas de aire \\
\hline Ciclón & Viento que gira alrededor de un centro de baja presión y avanza a menudo a grandes velocidades \\
\hline Meteorología & Ciencia que estudia las leyes que rigen los fenómenos que tienen lugar en la atmósfera \\
\hline Rayos & Una descarga eléctrica que se produce entre una nube y la tierra o entre dos nubes \\
\hline Lluvia & $\begin{array}{l}\text { Agua que procede de la atmósfera y que en forma líquida o sólida y se deposita sobre la super- } \\
\text { ficie de la tierra. }\end{array}$ \\
\hline Ciclo & Estado de la materia en que las moléculas que lo componen quedan poco ligadas entre ellas \\
\hline Cúmulus & $\begin{array}{l}\text { Nube blanca de formas redondeadas con muchas protuberancias en la parte superior y plano a } \\
\text { la inferior }\end{array}$ \\
\hline Vapor & Dicho de la fase gaseosa de una sustancia \\
\hline Clima & Condiciones atmosféricas (temperatura, lluvia, viento, etc.) determinando (incompleto) \\
\hline Sol & Estrella en torno al cual giran los planetas del sistema solar \\
\hline Metro & Del latín Metrum: medida, instrumento de medida \\
\hline Atmósfera & Es una capa de gases que rodea toda la tierra \\
\hline Nieve & $\begin{array}{l}\text { Pequeños cristales de glas provenientes de la congelación del agua de las nubes y que caen } \\
\text { sobre la tierra. }\end{array}$ \\
\hline
\end{tabular}

Tabla 6. Ejemplos de definiciones.

\subsection{Análisis en categorías de palabras}

Categorizar objetos o eventos es encontrar elementos comunes; así como categorizar las palabras de las listas, es considerar lo que tienen de equivalentes, lo que comparten como características comunes. La categorización está implicada en todas las actividades cognitivas, desde las más simples a las más complejas: identificar, comparar, designar, representar relaciones abstractas, memorizar, recordar, aprender, etc. (Cèbe y Goigoux, 2015). Por ello, la maestra organizó la categorización de 
las palabras entre los niños para ayudarlos a tomar conciencia de los criterios de la organización. En esta actividad, orientamos la categorización en términos de clases de palabras, básicamente nombres, verbos y adjetivos. Esta actividad resultó ser la más compleja: los alumnos no habían recibido formación gramatical durante los cursos anteriores y la actividad implicaba una reflexión metalingüística todavía muy incipiente en estas edades. Pero, las respuestas a esta tarea servirían para orientar futuras actuaciones: ¿llegarían los alumnos a categorizar las palabras del vocabulario?, ¿cuáles categorías resultarían mejor o peor solucionadas?

\begin{tabular}{|l|l|l|}
\hline \multicolumn{1}{|c|}{ Nombres } & \multicolumn{1}{|c|}{ Verbos } & \multicolumn{1}{c|}{ Adjetivos } \\
\hline $\begin{array}{l}\text { Autobús, autocar, antibiòtic, } \\
\text { excampions, exnuvi, taxista, } \\
\text { menjador, maquinista, } \\
\text { supermercat, nuvolositat, } \\
\text { meteoròleg, pescador, } \\
\text { entrenador, nevada, etc. }\end{array}$ & $\begin{array}{l}\text { Ballar, nedar, cantar, aletejar, } \\
\text { airejar, vesprejar, gatejar, } \\
\text { anteposar, etc. }\end{array}$ & $\begin{array}{l}\text { Nuvolat, congelat, plujós, } \\
\text { ventós, gelat, nevat, descongelat, } \\
\text { ràpidament, gelada, multicolor, } \\
\text { assolellat, nevada, etc. }\end{array}$ \\
\hline
\end{tabular}

Tabla 7. Ejemplos de definiciones.

La categorización se hizo basándose en la semejanza funcional. Los alumnos procedían argumentando por colocaciones paradigmáticas, con comentarios del tipo «una nubosidad, una nevada, entonces es nombre», o bien «es algo multicolor», es adjetivo. Es decir, agrupaban en categorías paradigmáticas los ítems que se comportaban de forma semejante.

\section{Conclusiones}

En este artículo hemos querido mostrar una manera en que la experiencia de las actividades de clase puede influir sobre el aprendizaje del vocabulario. Entre los aspectos referidos están la experiencia con uso de textos y de instrumentos de consulta (libros, enciclopedias, recursos digitales), dentro de una tarea de selección de vocabulario relacionado con el tema que se está estudiando. Usando esos instrumentos de consulta, los alumnos hicieron una colección de palabras, en listas de un rango entre 60 y 117 palabras, lo que sirvió como la «masa crítica» para el posterior trabajo lingüístico con el texto.

A partir de la inicial selección del conjunto de vocabulario, los alumnos pudieron realizar actividades de organización de las palabras seleccionadas y de comparación de su estructura en términos de raíces y afijos de las palabras. Este tipo de trabajo cognitivo sobre el input y la práctica de manipular, categorizar, comparar y establecer semejanzas ayuda evidentemente a la comprensión. Establecer una masa crítica de vocabulario temático permite a los alumnos sobreponerse a la dificultad con los textos académicos.

Las actividades que aquí se proponen responden tanto a la concepción de la importante relación entre el vocabulario y el texto, así como a la concepción de cómo se aprende el vocabulario. Hemos justificado el papel del vocabulario en la organización textual. Si el vocabulario de los textos académicos es técnico, abstracto, poco frecuente en el uso del alumno, esto contribuye a aumentar las dificultades de comprensión del texto. Por ello, es importante en la práctica pedagógica comenzar trabajando el vocabulario en tareas como las aquí propuestas de seleccionar, organizar y comparar las palabras. Estas actividades, juntos con la de manipular el vocabulario, se justifican 
también porque responden al proceso de aprendizaje lexical. En efecto, como se ha explicado, el aprendizaje del vocabulario no es solo sólo declarativo o proposicional, sino que también es un conocimiento procedimental. Por tanto, no se trata solamente de buscar el significado de las palabras sino de usarlas en situaciones y actividades diferentes.

La experiencia que hemos presentado se ha realizado en la clase de una escuela, con la participación activa de la profesora que, con nuestra orientación técnica, ha programado el tema del currículo integrando el conocimiento de ciencias naturales con conocimiento del lenguaje y la comprensión del texto. El presente artículo aporta la descripción de ese trabajo conjunto de coordinación entre orientadores y profesora con justificación teórica y aplicación en clase sobre el aprendizaje de vocabulario. Las actividades aquí propuestas concretan ese trabajo conjunto.

\section{Referencias bibliográficas}

Bates, E., y Goodman, J. (1997). On the inseparability of grammar and the lexicon: Evidence from acquisition, aphasia and real-time. Language and Cognitive Processes, 12(5/6), 507-584.

Beck, I. L., y McKeown, M. G., (2007). Increasing young low income children's oral vocabulary repertoires through rich and focused instruction. Elementary School Journal, 107(3), 251-271.

Bernárdez, E. (2010). El léxico como motor de la organización del texto. Revista de Investigación Lingüística, 13, 41-65.

Biber, D. (2006). University language: A corpus-based study of spoken and written registers. Philadelphia: John Benjamins.

Biemiller, A. (2009). Words worth teaching. Columbus, OH: SRA/McGraw-Hill.

Biemiller, A. (2012). Words for English-language learners. TESL Canada Journal/Eevue TESL du Canada, 201, 29, Special Issue 6, 198-203.

Biemiller, A., y Boote, C. (2006). An effective method for building meaning vocabulary in primary grades. Journal of Educational Psychology, 98(1), 44-62.

Booij, G. (2015). The structure of words. En J. Taylor (Ed.), The Oxford handbook of the word, p.157174. Oxford: Oxford University Press.

Bybee, J. (1998). The emergent lexicon. Papers from the Regional Meetings, Chicago Linguistic Society, 2, 421-435.

Calaque, E. (2004). Construction du lexique et construction des connaissances, Colloque AIRDF, Québec, www.colloqueairdf.fse.ulaval.ca/

Carlisle, J.E. (2003). Morphology matters in learning to read: a commentary. Reading Psychology, 24, 291-322.

Cèbe, S., y Goigoux, R. (2015). Vocabulaire et comprehension de textes: et si nous ne laissions à l'élève que la charge qui lui revient? Spirale: revue de recherches en éducation, ÉSPÉ LilleNord de France. Supports et pratiques d'enseignement: quels risques d'inégalités?, 119-136.

Chamorro, D., Barletta, N., y Mizuno, J. (2013). El lenguaje para enseñar y aprender las Ciencias Naturales: Un caso de oportunidades perdidas para la formación ciudadana. Revista Signos. Estudios de Lingüística, 46, 81, 3-28.

Chung, T.W., y Nation, P. (2003). Technical vocabulary in specialized texts. Reading in a Foreign Language, 15(2), 103-116.

Colé, P. (2011). Le développement du vocabulaire à lécole primaire: les apports de la dimension morphologique de la langue. Éduscol: Le vocabulaire et son enseignement. Recuperado de eduscol.educa-tion.fr/vocabulaire 
Corbin, D. (1991). Morphologie dérivationnelle et structuration du lexique. Sens et structures. Presses Universitaires de Lille.

Coxhead, A. (2000). A new academic word list. TESOL Quarterly 34.2, 213-238.

Crystal, D. (2015). The lure of words. En J. R. Taylor (Ed.), The Oxford handbook of the word, p. 23-28. Oxford: Oxford University Press.

Cunningham, A. E., y Stanovich, K. E. (1991). Tracking the unique effects of print exposure in children: Associations with vocabulary, general knowledge, and spelling. Journal of Educational Psychology, 83, 264-274.

David, J. (2000). Le lexique et son acquisition: aspects cognitifs et linguistiques. Le français aujourd'hui, 131, 31-40.

David, J. (2003). Construction du lexique et acquisition de la lecture. ONL: Les manuels de lecture.

Ferreiro, E. (2002). Escritura y oralidad: unidades, niveles de análisis y conciencia metalingüística. En E. Ferreiro (Comp.), Relaciones de (in)dependencia entre oralidad y escritura, p. 151-172. Barcelona: Gedisa Editorial.

Ferreiro, E., y Vernon, A. (1992). La distinción palabra/nombre en niños de 4 y 5 años. Infancia y Aprendizaje, 58, 15-28.

Fournier, J.-L. (2006) Les mots des riches, les mots des pauvres. Paris: Anne Carrière Édition.

García, J.N., y González, L. (2006). Diferencias en la conciencia morfológica, la escritura y el lenguaje, en función del desarrollo y el nivel educativo del niño. Psicothema, 18(2), 171-179.

Grossmann, F. (2011). Didactique du lexique : état des lieux et nouvelles orientations. Pratiques, 149-150, Recuperado de http:// pratiques.revues.org/1732; DOI : 10.4000/pratiques.1732

Halliday, M. A. K. (1989). Some grammatical problems in scientific English. Australian Review of Applied Linguistics. Supplement, 6, 13-37.

Harmon, J., Hedrick, W., y Wood, K. (2005). Research on vocabulary instruction in the content areas: Implications for struggling readers. Reading \& Writing Quarterly, 21, 261-280.

Hart, B., y Risley, T. R. (1999). The social world of children learning to talk. Baltimore, MD: Paul H. Brookes Publishing Co.

Lehmann, A. (2011). Idées reçues sur le lexique: un obstacle à l'enseignement du lexique dans les classes Éduscol: Le vocabulaire et son enseignement. Recuperado de eduscol.education.fr/vocabulaire

Lété, B. (2004). MANULEX: Le lexique des manuels scolaires de lecture. Implications pour l'estimation du vocabulaire des enfants de 6 à 11 ans. En E. Calaque y J. David (Eds.), Didactique du lexique: Contextes, démarches, supports , 241-257. Bruxelles: De Boeck.

Mel'cuk, I., Clas, A., y Polguere, A. (1995). Introduction à la lexicologie explicative et combinatoire. Paris: Duculot.

Mercer, N. (1995). Investigación sobre el conocimiento compartido. En G. Walford (Ed.), La otra cara de la investigación educativa, 59-78. Madrid: La Muralla.

Middleton, P. (2005). How to read a reading of a written poem. Oral Tradition, 20,1, 7-34.

Nagy, W. E., y Anderson, R. (1984). How many words are there in printed English schoolbooks? Reading Research Quarterly, 19(3), 304-330. doi:10.2307/747823

Nagy, W., Herman, P., y Anderson, R. (1985). Learning words from context. Reading Research Quarterly, 20, 223-253.

Nagy, W., y Townsend, D. (2012). Words as tools: Learning academic vocabulary as language acquisition. Reading Research Quarterly, 47(1), 91-108. doi: 10.1002/RRQ.011

Nation, I.S.P. (2001). Learning vocabulary in another language. Cambridge: Cambridge University Press. 


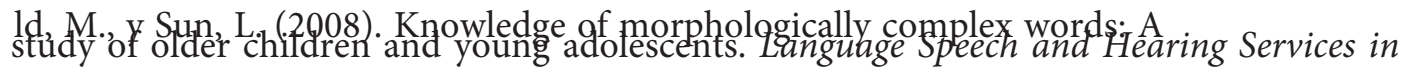

Nation, P. (2015). Which words do you need? En J. R, Taylor (Ed.), The Oxford handbook of the word, p. 568-580. Oxford: Oxford University Press.

Neuman, S. B., y Dwyer, J. (2011). Developing vocabulary and conceptual knowledge for low-income preschoolers: A design experiment. Journal of Literacy Research, 43(2) 103-129.

Nippold, M. A. (2007). Later language development: School-age children, adolescents, and young adults ( $3^{\text {rd }}$ ed.). Austin, TX: Pro-Ed.

Nippold, M., y Sun, L. (2008). Knowledge of morphologically complex words. A developmental study of older children and young adolescents. Language Speech and Hearing Services in Schools, 39, 365-373. doi: 10.1044/0161-1461(2008/034) · Source: PubMed.

Paveau, A-M. (2006). Chronique «linguistique». Leçon de vocabulaire (1), Le français aujourd'hui, $3,154,121-128$.

Peris Morant, A. (2012). Nominalizaciones deverbales: denotación y estructura argumental. Tesis doctoral, Universitat de Barcelona, Departament de Lingüística General

Petit, G. (2000). Didactique du lexique et problématique de l'unité lexicale: état d'une confusion. Le Français Aujourd'hui, 131, 53-62.

Reinking, D., y Bradley, B.A. (2004). Connecting research and practice using formative and design experiments. En N. K. Duke y M. H. Mallette (Eds.), Literacy research methodologies, p. 149-169. New York: Guilford.

Reinking, D., y Watkins, J. (2000). A formative experiment investigating the use of multimedia book reviews to increase elementary students' independent reading. Reading Research Quaterly, 35, 3, 384-419.

Rey-Debove, J. (1984). Le domaine de la morphologie lexicale. Cahiers de Lexicologie, 45, 3-19.

Rey-Debove, J (2004). Le Robert Brio. Analyse comparative des mots. Paris: Le Robert.

Sepúlveda, A., y Teberosky, A. (2008). Elaboración de listas a partir de textos y textos a partir de listas. Una actividad para aprender lenguaje escrito. Lectura y Vida, 29(4), 6-19.

Stanovitch, K.E. (1986), Matthew effect in reading: some consequences of individual differences in the acquisition of literacy. Reading Research Quarterly, 21, 360-406.

Svendro, J. (1997). La Grecia arcaica y clásica. La invencion de la lectura silenciosa. En G. Cavallo y R. Chartier (Eds.), Historia de la lectura en el mundo occidental, 57-94. Madrid: Taurus.

Waxman, S., y Markow, D. (1995). Words as invitations to form categories: Evidence from 12 to 13-month-old infants. Cognitive Psychology, 29, 257-302. 